\title{
Abdominal $\mathrm{CT}$ radiation dose optimization at Siriraj Hospital
}

Piyaporn Apisarnthanarak, M.D.

Chosita Buranont, M.D.

Chulaluck Boonma, B.Sc.

Sureerat Janpanich, RN.

Tarntip Suwatananonthakij, RN.

Atchariya Klinhom, RN.

Kobkun Muangsomboon, M.D.

Wanwarang Teerasamit, M.D.

Voraparee Suvannnarerg, M.D.

Pairash Saiviroonporn, Ph.D.

From Department of Radiology, Faculty of Medicine Siriraj Hospital, Mahidol University, Bangkok, Thailand.

Address correspondence to P.A. (e-mail: punpae159@gmail.com)

\section{Abstract}

Objective: To compare radiation dose and image quality between standard dose abdominal CT currently performed at our hospital and new low dose abdominal CT using various percentages $(0 \%, 10 \%, 20 \%$, and 30\%) of Adaptive Statistical Iterative Reconstruction (ASiR).

Materials and Methods: We prospectively performed low dose abdominal CT (30\% reduction of standard tube current) in 119 participants. The low dose CT images were post processed with four parameters $(0 \%, 10 \%, 20 \%$ and $30 \%)$ of ASiR. The volume CT dose index (CTDIvol) of standard and low dose CT were compared. Four experienced abdominal radiologists independently assessed the quality of low dose CT with aforementioned ASiR parameters using a 5-pointscale satisfaction score $(1=$ unacceptable, $2=$ poor, $3=$ average, $4=$ good, and $5=$ excellent image quality) by using prior standard dose CT as a reference of excellent image quality (5). Each reader selected the preferred ASiR parameter for each 
participant. The image noise of the liver and the aorta in all 5 (1 prior standard dose and 4 current low dose) image sets was measured.

Results: The mean CTDIvol of low dose CT was significantly lower than of standard dose CT $(7.17 \pm 0.08$ vs $12.02 \pm 1.61 \mathrm{mGy}, \mathrm{p}<0.001)$. The mean satisfaction scores for low dose CT with $0 \%, 10 \%, 20 \%$ and $30 \%$ ASiR were 3.95, $3.99,3.91$ and 3.87 , respectively with the ranges of 3 to 5 in all techniques. The preferred ASiR parameters of each participant randomly selected by each reader were varied, depending on the readers' opinions. The mean image noise of the aorta on standard dose CT and low dose CT with 0\%, 10\%, 20\%, and 30\% ASiR was $29.07,36.97,33.92,31.49$, and 29.11 , respectively, while the mean image noise of the liver was $24.60,30.21,28.33,26.25$, and 24.32 , respectively.

Conclusion: Low dose CT with $30 \%$ reduction of standard $\mathrm{mA}$ had acceptable image quality with significantly reduced radiation dose. The increment of ASiR was helpful in reducing image noise.

Keywords: Abdominal computed tomography, Abdominal CT, Radiation dose reduction, Iterative reconstruction, IR, Adaptive Statistical Iterative Reconstruction, ASiR.

\section{Introduction}

The image quality of abdominal computed tomography (CT) is one of the main factors for accurate CT interpretation. Unfortunately, the quality of CT scan increases in accordance with the high radiation exposure. This problem is a major concern among patients and radiologists worldwide. There were many proposed techniques of radiation dose reduction, such as minimizing the number of CT acquisitions and area coverage as necessary, reducing tube current, and decreasing peak kilovoltage[1,2]. However, increasing image noise and beamhardening artifacts were the unavoidable consequences. The CT vendors proposed many techniques for optimizing image quality of low dose CT scan. One widely accepted reconstruction technique was iterative reconstruction (IR) which 
provided less image noise than the conventional filtered back projection (FBP) reconstruction technique[3-5].

In our hospital, we continued to reduce CT radiation dose for patients' safety. At the same time, we needed to balance image quality for the sake of accurate CT interpretation. We instructed our residents and fellows to protocol each patient for a suitable number of $\mathrm{CT}$ acquisitions and area coverage. According to our abdominal CT standard protocol, we used the tube current of 400 and $340 \mathrm{~mA}$ for 64-slice and 256-slice CT scanners, respectively. With the new de-noising IR technique (Adaptive Statistical Iterative Reconstruction, ASiR), we recently performed the pilot study of 22 abdominal CT with radiation dose reduction, which achieved acceptable image quality with $30 \%$ reduction of our standard tube current, from 400 to be $260 \mathrm{~mA}$ on 64 -slice CT scanner and from 340 to be 210 $\mathrm{mA}$ on 256-slice CT scanner (standard $\mathrm{mA}$ multiplied with $\mathrm{mA}$ adjustment factor for $30 \%$ dose reduction of 0.66 and 0.63 for 64 -slice and 256-slice CT scanners, respectively). This current study with a larger number of study participants were prospectively performed for the purpose of comparing radiation dose and image quality between standard abdominal CT currently performed at our hospital and the new low dose abdominal CT with various parameters of IR techniques.

\section{Materials and methods}

\section{Study Designs and Participants}

This study was a prospective, single-centered study performed at a 2,200bed university hospital in central Thailand. This study was approved by our institutional review board with informed consents from all included participants. All participants were aged over 18 years old who were scheduled for contrast enhanced abdominal CT examinations at our department in January 2018. They had prior standard dose contrast enhanced abdominal CT available on our Picture Archiving and Communication System (PACS). One hundred and nineteen patients met the criteria and were recruited as our study population. The demographic data of each participant including gender and age were recorded by one of our investigators (CB). 


\section{CT Techniques}

\section{Standard Dose Abdominal CT}

The prior standard dose abdominal CT of our participants was routinely performed by four General Electric (GE) CT scanners including three 64-slice scanners (one LightSpeed VCT and two Discovery CT750 High Definition, GE Healthcare, Milwaukee, WI, USA) and one 256-slice scanner (Revolution CT, GE healthcare, Milwaukee, WI, USA). The CT of each participant was protocoled for a suitable number of CT acquisitions and area coverage. All participants were advised to hold their breath during the scan. The scan coverage included at least the upper abdominal area. The slice collimation was $1.25 \mathrm{~mm}$ (reconstructed at $7.0 \mathrm{~mm}$ ) for all scanners. There were varieties on the administration of oral and rectal contrasts according to each participant's suitable protocol. All participants underwent precontrast and postcontrast studies, before and after a bolus intravenous injection of nonionic iodinated contrast agent ( $2 \mathrm{~mL}$ per $\mathrm{kg}$ body weight), followed by 20 $\mathrm{mL}$ of water via a power injector at a rate of $3 \mathrm{~mL} / \mathrm{second}$. Each participant had at least a portovenous phase with an 80 -second delay for postcontrast study. An additional arterial phase at 35 to 40 -second delay or delayed phase at 5 to 10 minute delay were obtained in some participants as necessary. The peak kilovoltage was fixed at $120 \mathrm{kVp}$ for all scanners. The tube current based on our standard protocol was 400 and $340 \mathrm{~mA}$ for 64-slice and 256-slice CT scanners, respectively. The rotation time was 0.5 second for all scanners. The pitch was 1.375:1 and 0.992:1 for for 64-slice and 256-slice CT scanners, respectively. All images were reconstructed with the standard FBP techniques and sent to PACS for subsequent reviews.

\section{Low Dose Abdominal CT}

The low dose abdominal CT of the study participants were performed by three GE CT scanners including two 64-slice scanners (Discovery CT750 High Definition, GE Healthcare, Milwaukee, WI, USA) and one 256-slice scanner (Revolution CT, GE healthcare, Milwaukee, WI, USA). Our remote 64-slice CT scanner (LightSpeed VCT, GE healthcare, Milwaukee, WI, USA) did not have IR de-noising technique for improving image quality; therefore, it was not included 
in the performance of low dose abdominal CT. The CT scanners for standard and low dose CT of each participant were not fixed to be the same scanner. The CT of each participant was protocoled for a suitable number of CT acquisitions and area coverage (at least cover the upper abdominal area). The scan techniques were the same as described in prior standard dose abdominal CT section except for the tube current on the portovenous phase which was reduced for $30 \%$; from 400 to be $260 \mathrm{~mA}$ on 64 -slice CT scanners and from 340 to $210 \mathrm{~mA}$ on 256 -slice CT scanner (standard $\mathrm{mA}$ multiplied with $\mathrm{mA}$ adjustment factor for $30 \%$ dose reduction of 0.66 and 0.63 for 64-slice and 256-slice CT scanners, respectively). The other phases used the standard tube current ( 400 and $340 \mathrm{~mA}$ for 64 -slice and 256-slice CT scanners, respectively). We chose to study only the portovenous phase because most abdominal organs had homogeneous enhancement on this phase. It was easy for radiologists to evaluate the CT image quality.

The IR technique specific for our GE CT scanners (Adaptive Statistical Iterative Reconstruction, $\mathrm{ASiR}$ ) was applied by blending with the conventional FBP on low dose portovenous phase images by post-processing at a CT workstation with the range of $0 \% \mathrm{ASiR}$ (with 100\% FBP), 10\% ASiR (with 90\% FBP), 20\% ASiR (with $80 \% \mathrm{FBP}$ ) and $30 \% \mathrm{ASiR}$ (with 70\% FBP). With these reconstruction techniques, each participant had four sets of low dose CT images on the portovenous phase sent to PACS for subsequent reviews.

For a parameter of radiation dose comparison, we selected volume CT dose index (CTDIvol) instead of the dose length product (DLP). The DLP would depend on the length of scan which varied in the participants due to the difference in area coverage and the number of $\mathrm{CT}$ acquisitions.

The details of CT scanners, study dates, and CTDIvol of each participant's prior standard dose abdominal CT and current low dose abdominal CT were recorded by one of our investigators (CB). The time interval between the two studies was calculated. 


\section{CT Analysis}

For qualitative assessment of low dose abdominal CT, four board-certified, fellowship-trained abdominal radiologists (PA, KM, WT, and VS with 22, 22, 16, and 13 years of experience in abdominal CT evaluation) separately reviewed 5 sets of abdominal CT images ( 1 set of prior standard dose portovenous phase images; and 4 sets of low dose portovenous phase images with $0 \%, 10 \%, 20 \%$, and $30 \% \mathrm{ASiR}$ ) of each participant. All readers were not blinded to the percentage of applied ASiR. They graded the image quality of each low dose CT set by using a 5-point-scale satisfaction score on a visual scale as follows:

1: Unacceptable image quality, unable to interpret

2: Poor image quality, interfering with interpretation

3: Average image quality, possible interpretation

4: Good image quality

5: Excellent image quality

The satisfaction score was given by using each participant's prior standard dose CT images as a reference of excellent image quality (5). The satisfaction score of 3 to 5 were acceptable for CT interpretation. Each reader selected the preferred image set from 4 sets of low dose abdominal CT for each participant.

For quantitative assessment of abdominal CT, the image noise of the aorta and the liver was measured on either routine standard dose CT images or 4 sets of low dose CT images by one of our investigators (CB) on a CT workstation (Advantage workstation AW 4.6, GE healthcare, Milwaukee, WI, USA). The image noise was measured by drawing circular region of interests (ROIs) at 4 locations (one aortic and 3 hepatic regions) on a $1.25-\mathrm{mm}$ slice portovenous image at the same locations and levels of these 5 image sets. For image noise of the aorta, the ROI was drawn at least $1 / 3$ area of aortic lumen $\left(60-90 \mathrm{~mm}^{2} \pm 5 \mathrm{~mm}^{2}\right)$ at the most central part to avoid calcified plaque at the aortic wall. Three hepatic ROIs $\left(100-150 \mathrm{~mm}^{2}\right.$ $\pm 5 \mathrm{~mm}^{2}$ ) were routinely applied on the left lobe, the anterior right lobe, and the posterior right lobes (Figure 1). In patients with prior hepatic surgery, the ROIs were placed in three different locations in the remaining hepatic areas. The hepatic ROIs were placed at the homogenous enhancing hepatic areas avoiding vessels, 
bile ducts, hepatic lesions, calcifications and surgical materials. The mean image noise of each liver was calculated from these 3 hepatic ROIs of image noise.

\section{Statistical Analysis}

The demographic data of participants, CT scanners, time interval between CT studies, the image quality (satisfaction scores, readers' preferred ASiR parameters, image noise) and CTDIvol of low dose and standard dose CT were presented as number (\%), mean (standard deviation, SD), median, and range. Paired t-test was used to compare mean CTDIvol between standard dose CT and low dose CT. Multivariate analysis with Bonferroni adjustment for a pairwise comparison was applied to compare mean satisfaction scores, mean image noise of the aorta and the liver among different ASiR parameters.

All statistical data analyses were performed by using PASW 18.0 (SPSS Inc., Chicago, IL, USA). A 2 -sided p-value of less than or equal to 0.05 was considered a statistical significance. 

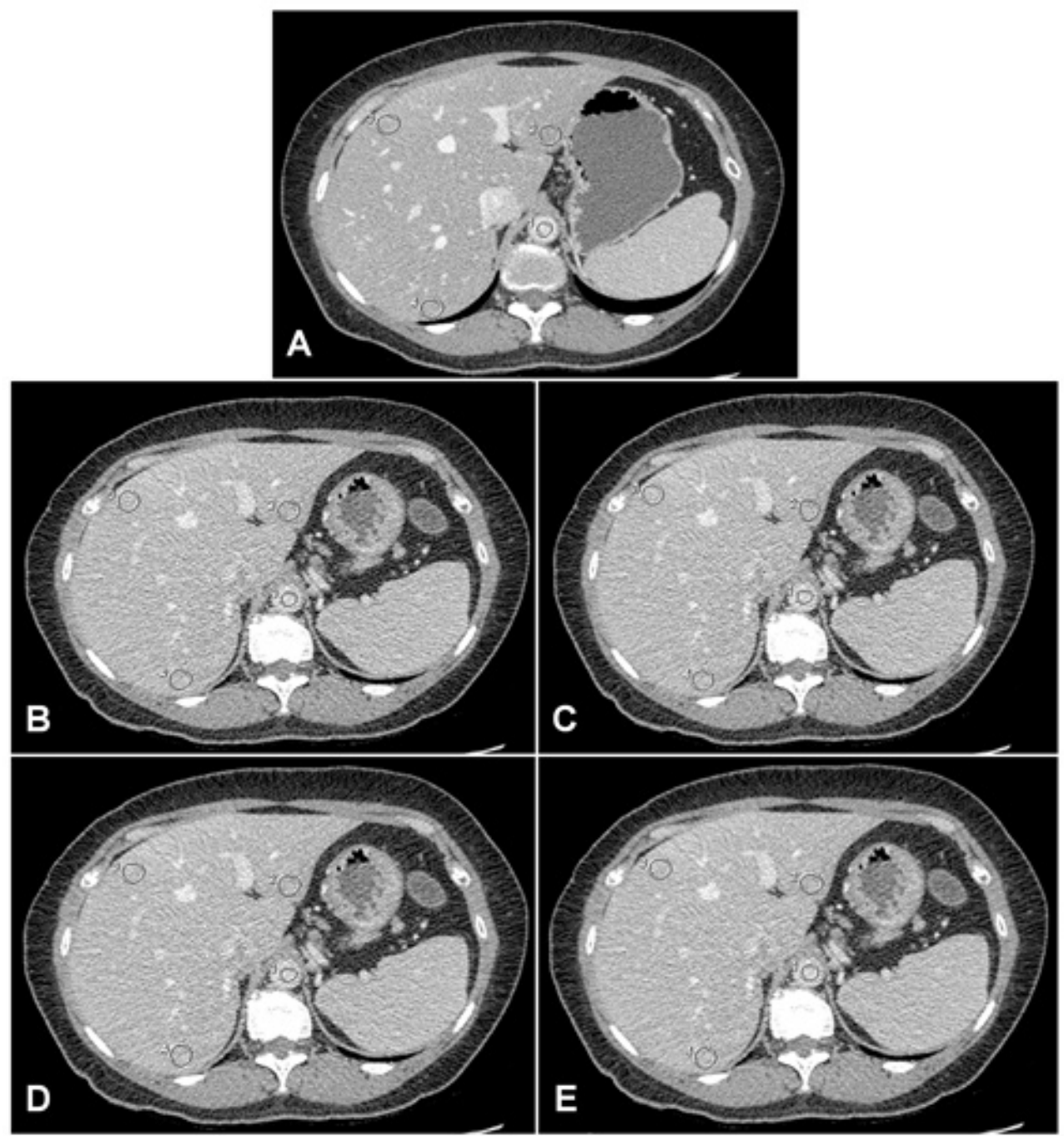

Figure 1. The image noise measurement of the aorta (1 ROI) and the liver (3 ROIs at the left lobe, the anterior right lobe and the posterior right lobe) on the 5 image sets (A-E)

A: Prior standard dose abdominal CT,

B-E: The low dose abdominal CT with 0\% ASiR (B), 10\% ASiR (C), 20\%

ASiR (D) and 30\% ASiR (E).

The ROI were positioned at the same locations and levels for all 5 image sets. 


\section{Results}

\section{Participants}

One hundred and nineteen participants in this study included 50 (42.0\%) men and $69(58.0 \%)$ women. The mean age (SD) of the participants at the time of low dose CT scan was 59.2 (14.3) years with the range of $18-88$ years.

\section{CT Techniques}

The standard dose abdominal CT of $80(67.2 \%)$ and 39 (32.8\%) participants were performed by 64 -slice and 256-slice scanners, respectively. The low dose abdominal CT of $78(65.5 \%)$ and 41 (34.5\%) participants were performed by 64-slice and 256-slice scanners, respectively. The time interval between the two studies ranged from 6 to 422 days (median 161 days).

The mean CTDIvol (SD) of low dose CT was significantly lower than of standard dose CT, 7.17 (0.08) vs 12.02 (1.61) $\mathrm{mGy}, \mathrm{p}<0.001$.

\section{CT Analysis}

For qualitative assessment, the satisfaction score of low dose abdominal CT with $4 \mathrm{ASiR}$ parameters graded by 4 readers ranged from 3 to 5 , which were all acceptable for CT interpretation. The mean satisfaction scores of low dose abdominal CT with 4 ASiR parameters were summarized in Table 1. The preferred ASiR parameters applied to low dose CT of each participant randomly selected by each reader were varied, depending on the readers' opinions (Table 2).

For quantitative assessment, the image noise of the aorta and the liver on standard dose $\mathrm{CT}$ and low dose $\mathrm{CT}$ with $4 \mathrm{ASiR}$ parameters was summarized in Table 3. The image noise of the aorta and the liver was significantly increased on low dose CT with $0 \% \mathrm{ASiR}$ compared to prior standard dose CT. With the consecutive increment of $\mathrm{ASiR}$, the image noise sequentially decreased. There was no difference between the mean image noise of the aorta and the liver on low dose CT with $30 \%$ ASiR compared to prior standard dose CT $(\mathrm{p}=1.000)$ (Table 4). 
Table 1. The satisfaction scores of low dose abdominal CT with 0\%, 10\%, 20\%, and $30 \%$ ASiR parameters by 4 readers.

\begin{tabular}{lllll}
\hline & \multicolumn{4}{c}{ Mean Satisfaction Score (SD) of Low Dose Abdominal CT } \\
\cline { 2 - 5 } & 0\% ASiR & 10\% ASiR & 20\% ASiR & 30\% ASiR \\
\hline Reader 1 & $4.22(0.56)$ & $4.39(0.54)$ & $4.34(0.48)$ & $4.35(0.48)$ \\
Reader 2 & $4.01(0.09)$ & $4.01(0.09)$ & $4.01(0.09)$ & $4.01(0.09)$ \\
Reader 3 & $3.66(0.68)$ & $3.80(0.65)$ & $3.82(0.63)$ & $3.93(0.55)$ \\
Reader 4 & $3.92(0.63)$ & $3.76(0.58)$ & $3.45(0.52)$ & $3.20(0.48)$ \\
All readers & $3.95(0.37)$ & $3.99(0.34)$ & $3.91(0.31)$ & $3.87(0.28)$ \\
\hline
\end{tabular}

Note: There were significant statistical differences between $0 \%$ ASiR vs $30 \%$ ASiR $(p=0.001)$ $10 \%$ ASiR vs $20 \%$ ASiR $(p<0.001)$ $10 \%$ ASiR vs 30\% ASiR $(p<0.001)$

Table 2. The preferred ASiR parameters applied to low dose CT selected by 4 readers.

\begin{tabular}{|lccccc}
\hline & \multicolumn{2}{c}{ Number of The Preferred Low Dose Abdominal CT (\%) } & \multirow{2}{*}{ Total } \\
\cline { 2 - 5 } & 0\% ASiR & 10\% ASiR & 20\% ASiR & 30\% ASiR & AS \\
\hline Reader 1 & $42(35.3)$ & $23(19.3)$ & $40(33.6)$ & $14(11.8)$ & $119(100.0)$ \\
Reader 2 & $1(0.8)$ & $2(1.7)$ & $87(73.1)$ & $29(24.4)$ & $119(100.0)$ \\
Reader 3 & $0(0.0)$ & $0(0.0)$ & $0(0.0)$ & $119(100.0)$ & $119(100.0)$ \\
Reader 4 & $110(92.4)$ & $9(7.6)$ & $0(0.0)$ & $0(0.0)$ & $119(100.0)$
\end{tabular}


Table 3. The image noise of the aorta and the liver on standard dose CT and low dose CT with 4 ASiR parameters.

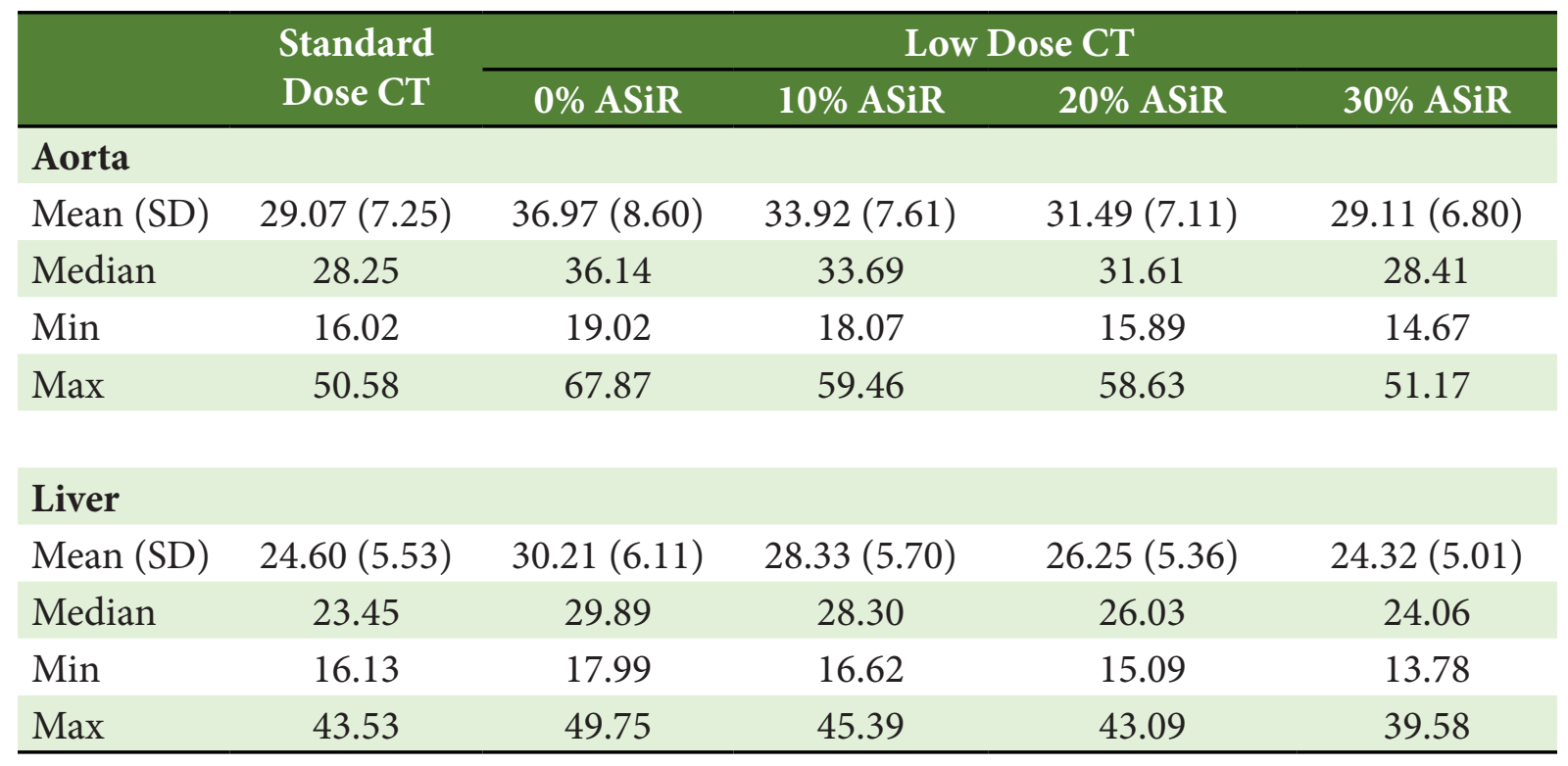

Table 4. The difference of mean image noise of the aorta and the liver on low dose CT with 4 ASiR parameters compared to standard dose CT.

\begin{tabular}{|c|c|c|c|}
\hline & $\begin{array}{l}\text { Difference (SD) of Mean Image Noise } \\
\text { Compared to Standard Dose CT }\end{array}$ & $\underline{p \text {-Value }}$ & $95 \% \mathrm{CI}$ \\
\hline \multicolumn{4}{|l|}{ Aorta } \\
\hline $0 \%$ ASiR & $7.90(0.74)$ & $<0.001$ & $5.79,10.01$ \\
\hline 10\% ASiR & $4.86(0.66)$ & $<0.001$ & $2.96,6.76$ \\
\hline 20\% ASiR & $2.42(0.66)$ & 0.004 & $0.54,4.30$ \\
\hline 30\% ASiR & $0.04(0.66)$ & 1.000 & $-1.83,1.92$ \\
\hline \multicolumn{4}{|l|}{ Liver } \\
\hline $0 \%$ ASiR & $5.61(0.51)$ & $<0.001$ & $4.15,7.06$ \\
\hline $10 \% \mathrm{ASiR}$ & $3.73(0.46)$ & $<0.001$ & $2.41,5.06$ \\
\hline $20 \%$ ASiR & $1.65(0.46)$ & 0.005 & $0.34,2.96$ \\
\hline 30\% ASiR & $-0.28(0.45)$ & 1.000 & $-1.58,1.01$ \\
\hline
\end{tabular}




\section{Discussion}

According to the major concern of $\mathrm{CT}$ radiation exposure, the $\mathrm{CT}$ vendors proposed several new CT reconstruction techniques to optimize the image quality, allowing the radiologists to continue decreasing CT radiation dose for the patients' safety while still achieving the diagnostic confidence. The IR is one of the new methods of image reconstruction that has been developed in the last decade. Each IR technique is unique for each CT vendor, i.e., ASiR for GE healthcare, adaptive iterative dose reconstruction (AIDR 3D) for Toshiba Medical Systems, iDose for Philips Healthcare, and sinogram-affirmed iterative reconstruction (SAFIRE) for Siemens Healthcare. At our hospital, all CT scanners were GE scanners; therefore, we used ASiR as our IR technique to blend with the conventional FBP technique to optimize the image quality of low dose CT. The objective of this study was to assess the suitable amount of ASiR applied with our low dose CT (30\% dose reduction from our standard dose), either the qualitative aspect (radiologists' satisfaction and their preferred ASiR parameter) or the quantitative aspect (image noise).

In our study, low dose CT scan by using 30\% $\mathrm{mA}$ reduction provided significantly lower radiation dose compared to standard dose CT. The quality of all low dose CT images was acceptable for interpretation. There were many previous studies assessing the imaging quality obtained from low dose CT with IR techniques[6-10], showing that IR technique helped optimize the image quality by reducing image noise and provided similar image quality as standard dose CT. The satisfaction score in our study did not sequentially increase along with the increment of ASiR and the selected preferred ASiR parameters were varied by our radiologists' opinions. We assumed that the images with high percentage of ASiR provided smooth image appearances with less sharp borders. This was the reported major drawback of the IR technique[6]. Some of our radiologists were possibly familiar with a relatively noisy image with sharp borders derived from the conventional FBP technique.

As expected, the image noise of the aorta and the liver was significantly increased on low dose CT with $0 \%$ ASiR compared to standard dose CT. With the 
consecutive increment of ASiR, the image noise sequentially decreased until there was no difference between low dose CT with 30\% ASiR compared to standard dose CT. However, in some participants with cirrhosis, ascites or diffuse subcutaneous edema, the image noise was significantly high despite the addition of $30 \% \mathrm{ASiR}$ (Figure 2). We postulated that the patients with these conditions were not suitable for low dose CT.

There were several limitations of our study. First, there were variables in our CT scanners. Although they were all GE scanners, most were 64-slice scanners and one was a 256-slice scanner. Of which, some CT parameters (i.e. $\mathrm{mA}$ and pitch) were not the same. Plus, the CT scanners for standard and low dose CT of each participant were not necessarily the same scanners. Second, the time interval between prior standard dose CT and subsequent low dose CT ranged from 6 to 422 days (median 161 days). With such a long interval, there would be some changes in patient's conditions which would affect the image quality. The new study with a shorter time interval should be designed. Third, our radiologists were not blinded to the percentage of applied ASiR. Fourth, image noise was measured on a 1.25 $\mathrm{mm}$ slice portovenous image of each image set. Actually, image noise should be measured by choosing 3-5 consecutive CT slices and the noise should be average for the statistical accuracy. Finally, we evaluated only the image quality of low dose CT, but did not evaluate the ability to detect lesion or diagnostic performance. To evaluate the diagnostic performances between low dose CT and standard dose CT, these 2 studies need to be performed on the same date and almost the same acquisition phase. These will inevitably increase radiation dose received by the participants.

In conclusion, low dose CT with $30 \%$ reduction of standard $\mathrm{mA}$ had acceptable image quality with significantly reduced radiation dose. The increment of ASiR technique was helpful in reducing image noise. 


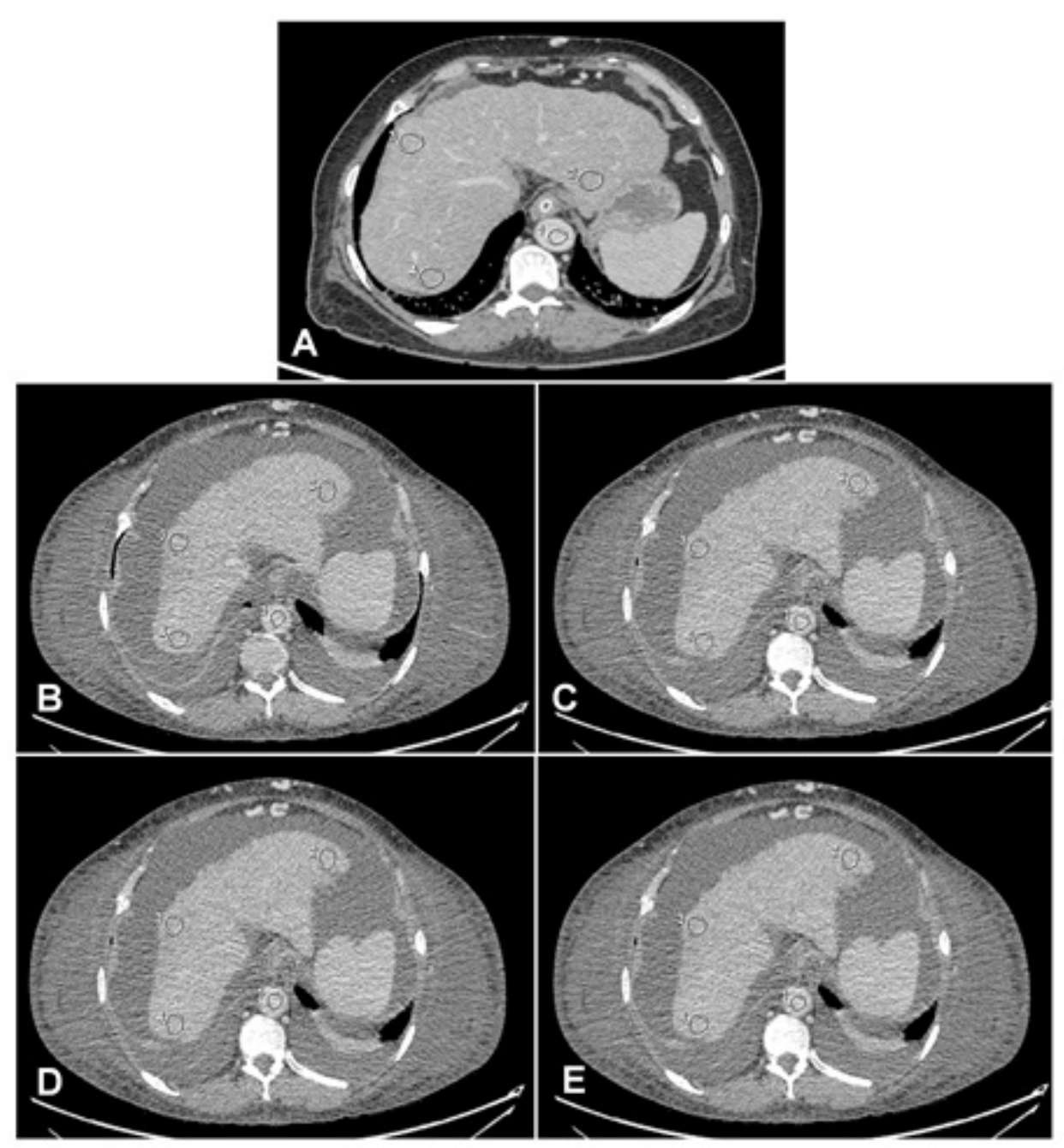

Figure 2. Poor image quality on low dose abdominal CT due to ascites and subcutaneous edema. Axial portovenous abdominal CT of a 58-year-old female with cirrhosis and portal hypertension $(A-E)$

A: Prior standard dose abdominal CT,

$B$-E: The low dose abdominal CT with 0\% ASiR (B), 10\% ASiR (C), 20\% ASiR

(D) and $30 \% \mathrm{ASiR}(\mathrm{E})$.

The time interval between the two studies was 295 days. The mean satisfaction score for ASiR $0 \%, 10 \%, 20 \%$ and $30 \%$ were 3.5, 3.5, 3.5 and 3.25, respectively. The mean liver image noise for standard dose, ASiR 0\%, 10\%, 20\% and 30\% were 19.05, 45.21, 40.10, 38.11 and 35.6, respectively. Note the development of ascites and subcutaneous edema dramatically affected the image quality. 


\section{References}

1. Hara AK, Wellnitz CV, Paden RG, Pavlicek W, Sahani DV. Reducing body $\mathrm{CT}$ radiation dose: beyond just changing the numbers. AJR Am J Roentgenol 2013;201:33-40. doi: 10.2214/AJR.13.10556.

2. Tamm EP, Rong XJ, Cody DD, Ernst RD, Fitzgerald NE, Kundra V. Quality initiatives: CT radiation dose reduction: how to implement change without sacrificing diagnostic quality. RadioGraphics 2011;31:1823-32. doi: 10.1148/rg.317115027.

3. Patino M, Fuentes JM, Singh S, Hahn PF, Sahani DV. Iterative reconstruction techniques in abdominopelvic CT: technical concepts and clinical implementation. AJR Am J Roentgenol 2015;205:W19-31. doi: 10.2214/ AJR.14.13402.

4. Willemink MJ, de Jong PA, Leiner T, de Heer LM, Nievelstein RA, Budde RP, et al. Iterative reconstruction techniques for computed tomography Part 1: technical principles. Eur Radiol 2013;23:1623-31. doi: 10.1007/s00330-012$2765-\mathrm{y}$.

5. Higaki T, Nakamura Y, Fukumoto W, Honda Y, Tatsugami F, Awai K. Clinical application of radiation dose reduction at abdominal CT. Eur J Radiol 2019;111:68-75. doi: 10.1016/j.ejrad.2018.12.018.

6. Mitsumori LM, Shuman WP, Busey JM, Kolokythas O, Koprowicz KM. Adaptive statistical iterative reconstruction versus filtered back projection in the same patient: 64 channel liver CT image quality and patient radiation dose. Eur Radiol 2012;22:138-43. doi: 10.1007/s00330-011-2186-3.

7. Chang W, Lee JM, Lee K, Yoon JH, Yu MH, Han JK, et al. Assessment of a model-based, iterative reconstruction algorithm (MBIR) regarding image quality and dose reduction in liver computed tomography. Invest Radiol 2013;48:598-606. doi: 10.1097/RLI.0b013e3182899104. 
8. Singh S, Kalra MK, Gilman MD, Hsieh J, Pien HH, Digumarthy SR, et al. Adaptive statistical iterative reconstruction technique for radiation dose reduction in chest CT: a pilot study. Radiology 2011;259:565-73. doi: 10.1148/ radiol.11101450.

9. Gervaise A, Osemont B, Louis M, Lecocq S, Teixeira P, Blum A. Standard dose versus low-dose abdominal and pelvic CT: comparison between filtered back projection versus adaptive iterative dose reduction 3D. Diagn Interv Imaging 2014;95:47-53. doi: 10.1016/j.diii.2013.05.005.

10. Singh S, Kalra MK, Hsieh J, Licato PE, Do S, Pien HH, et al. Abdominal CT: comparison of adaptive statistical iterative and filtered back projection reconstruction techniques. Radiology 2010;257:373-83. doi: 10.1148/ radiol.10092212. 